\title{
Ultrafine heat-induced structural perturbations of bone mineral at the individual nanocrystal level
}

M. Verezhak ${ }^{1,2}$, E. F. Rauch ${ }^{3}$, M. Véron ${ }^{3}$, C. Lancelon-Pin ${ }^{4}$, J.-L. Putaux ${ }^{4}$, M. Plazanet $^{1}$ and

A. Gourrier ${ }^{1 *}$

1 Univ. Grenoble Alpes, CNRS, LIPhy, 38000 Grenoble, France

2 Paul Scherrer Institut, 5232 Villigen PSI, Switzerland

3 Univ. Grenoble Alpes, CNRS, SIMAP, 38000 Grenoble, France

4 Univ. Grenoble Alpes, CNRS, CERMAV, 38000 Grenoble, France

* Corresponding author: aurelien.gourrier@univ-grenoble-alpes.fr; +33 476514729

\begin{abstract}
:
The nanoscale characteristics of the mineral phase in bone tissue such as nanocrystal size, organization, structure and composition have been identified as potential markers of bone quality. However, such characterization remains challenging since it requires combining structural analysis and imaging modalities with nanoscale precision. In this paper, we report the first application of automated crystal orientation mapping using transmission electron microscopy (ACOM-TEM) to the structural analysis of bone mineral at the individual nanocrystal level. By controlling the nanocrystal growth of a cortical bovine bone model artificially heated up to $1000{ }^{\circ} \mathrm{C}$, we highlight the potential of this technique. We thus show that the combination of sample mapping by scanning and the crystallographic information derived from the collected electron diffraction patterns provides a more rigorous analysis of the mineral nanostructure than standard TEM. In particular, we demonstrate that nanocrystal orientation maps provide valuable information for dimensional analysis. Furthermore, we show that ACOM-TEM has sufficient sensitivity to distinguish between phases with close crystal structures and we address unresolved questions regarding the existence of a hexagonal to monoclinic phase transition induced by heating. This first study therefore opens new perspectives in bone characterization at the nanoscale, a daunting challenge in the biomedical and archaeological fields, which could also prove particularly useful to study the mineral characteristics of tissue grown at the interface with biomaterials implants.
\end{abstract}

This document is the accepted manuscript version of the following article: Verezhak, M., Rauch, E. F., Véron, M., Lancelon-Pin, C., Putaux, J. L., Plazanet, M., \& Gourrier, A. (2018). Ultrafine heat-induced structural perturbations of bone mineral at the individual nanocrystal leve1. Acta Biomaterialia, 73, 500-508. https://doi.org/10.1016/j.actbio.2018.04.004 
Keywords: Bone; mineral nanocrystals; hydroxyapatite; TEM; electron diffraction; heating effects.

\section{Introduction}

Bone tissue is a biological nanocomposite material essentially composed of hydrated collagen fibrils of $\sim 100 \mathrm{~nm}$ in diameter and up to several microns in length, reinforced by platelet-shaped nanocrystals of calcium phosphate apatite of $\sim 3 \times 25 \times 50 \mathrm{~nm}^{3}$ in size [1]. These mineralized fibrils constitute the building blocks of bone tissue, and their specific arrangement is known to depend primarily on the dynamics of the formation and repair processes. Since these cellular processes can occur asynchronously in space and time, the mineralized fibrils adopt a complex hierarchical organization [1], which has been shown to be a major determinant of the macroscopic biomechanical properties [2]. Extensive research programs are therefore currently focused on bone ultrastructure for biomedical diagnoses or tissue engineering applications.

However, structural studies at the most fundamental scales remain challenging due to the technical difficulties imposed by nanoscale measurements and by the tissue heterogeneity. Nevertheless, as a natural extension of bone mineral density (BMD) analysis, an important marker in current clinical studies, the following key characteristics of the mineral nanocrystals have been identified as potential markers of age and diseases: chemical composition, crystallinity vs disorder, crystal structure, size, shape and orientation [3-4]. Recent progress in the field showed that in order to obtain a deeper medical insight into the mechanisms of bone function, several such parameters need to be combined and correlated to properties at larger length scales [5]. Interestingly, from a totally different point of view, the archaeological community has drawn very similar conclusions concerning nanoscale studies for the identification, conservation and restoration of bone remains and artifacts [6].

From a materials science perspective, this is a well identified challenge in the analysis of heterogeneous nanostructured materials. Yet, technically, a major difficulty stems from the fact that most of the identified nanostructural bone markers require individual measurements on dedicated instruments which are generally difficult to combine in an integrative approach.

One 'gold standard' in nanoscale bone characterization is X-ray diffraction (XRD), which allows determining atomic-scale parameters averaged over the total volume illuminated by the X-ray beam. An important result from XRD studies conducted with laboratory instruments is that the bone mineral phase has, on average, a poorly crystalline apatite 
structure which, to a certain extent, is induced by a high fraction of carbonate substitutions [7]. Such studies enabled localization of a substantial number of elements other than calcium and phosphorus present in bone via ionic substitutions [8], which can lead to serious pathological conditions, e.g. skeletal fluorosis [9]. When an average description of bone properties is insufficient, synchrotron X-ray beams focused to a typical diameter of $0.1-10$ $\mu \mathrm{m}$ [10-12] operated in a scanning mode allows mapping the microstructural heterogeneities. However, this remains intrinsically an average measurement and the current instrumentation limits prevent any analysis at the single mineral nanocrystal level.

Transmission electron microscopy (TEM) is a second 'gold standard' in bone characterization at the nanoscale. In high resolution mode, it allows reaching sub-angstrom resolution [13] and therefore provides atomic details of the crystals. This increased resolution comes at the cost of the image field of view, which may not provide representative results due to the tissue heterogeneity. This limitation can partly be alleviated in scanning mode, which is more adapted to the collection of a large amount of data for statistical usage. In particular, for the process known as Automated Crystal Orientation Mapping (ACOM-TEM) [14], diffraction patterns are systematically acquired while the electron beam is scanning micronsized areas, such that the structural parameters of hundreds of individual nanocrystals may be characterized and used to reconstruct orientation maps with a nanometer spatial resolution.

To our best knowledge, the present study is the first reported use of the ACOM-TEM method to analyze mineral nanocrystals in bone tissue. To demonstrate the potential of this technique for bone studies, a test object is required which structure should be as close as possible to native bone while offering a wide range of nanocrystal dimensions. Heated bone provides an ideal model for such purposes, ensuring a tight control over the nanocrystal size by adjusting the temperature. This system was extensively studied in archeological and forensic contexts. Upon heating to $100-150{ }^{\circ} \mathrm{C}$, bone is progressively dehydrated [15] and collagen is considered to be fully degraded at $\sim 400{ }^{\circ} \mathrm{C}[16,17]$. Most X-ray studies concluded an absence of mineral crystal structure modifications before $400{ }^{\circ} \mathrm{C}$, while a rapid crystal growth has been reported at $\sim 750{ }^{\circ} \mathrm{C}[18-20]$. In a recent study we provided evidence that the mineral nanocrystals increase in size and become more disorganized at temperatures as low as $100{ }^{\circ} \mathrm{C}$ [21]. In addition, many debates remain open concerning the nature of a postulated high temperature phase transition, the co-existence of different crystallographic phases, as well as the presence of ionic defects above and below the critical temperature of $T_{c r}=750{ }^{\circ} \mathrm{C}$ [22]. The heated bovine cortical bone model therefore presents two main advantages to assess 
the potential of ACOM-TEM: 1) the possibility to fine-tune the mineral nanocrystal size upon heating and 2) the existence of a phase transition at high temperatures.

Using a set of bovine cortical bone samples in a control state and heated at eight temperatures ranging from 100 to $1000{ }^{\circ} \mathrm{C}$, we show that ACOM-TEM provides enough sensitivity to probe fine crystalline modifications induced by heating; in particular, nanocrystal growth, subtle changes in stoichiometry and space group. Those results provide new insight into the detailed effects of heating on bone and validate the use of ACOM-TEM for fundamental studies of the nanoscale organization of bone tissue in different contexts.

\section{Materials and methods}

\subsection{Sample preparation}

A bovine femur was obtained from the local slaughterhouse (ABAG, FontanilCornillon, France). The medial cortical quadrant of a femoral section from the mid-diaphysis was extracted with a high precision circular diamond saw (Mecatome T210, PRESI) and fixed in ethanol $70 \%$ for 10 days (supplementary information, Fig. S1). Nine $2 \times 2 \times 10 \mathrm{~mm}^{3}$ blocks were cut in the longitudinal direction and subsequently dehydrated (48 hours in ethanol $70 \%$ and $100 \%$ ) and slowly dried in a desiccator. One block was used as a control, while the others were heated to eight temperatures: $100,200,300,400,600,700,800$ and $1000{ }^{\circ} \mathrm{C}$ for $10 \mathrm{~min}$ in vacuum $\left(10^{-2} \mathrm{mbar}\right)$ inside quartz tube and cooled in air. The temperature precision of the thermocouple was $\sim 2-3{ }^{\circ} \mathrm{C}$ and the heating rate was $\sim 30-40{ }^{\circ} \mathrm{C} / \mathrm{min}$. The heating process resulted in color change, as shown in Fig. S2 of supplementary information.

The samples were then embedded in poly-methyl methacrylate (PMMA) resin following the subsequent steps: impregnation, inclusion and solidification. For impregnation, a solution of methyl methacrylate (MMA) was purified by aluminum oxide and a solution of MMA was prepared with dibutyl phthalate in a 4:1 proportion (MMA1). The samples were kept at $4{ }^{\circ} \mathrm{C}$ in MMA1 for 5 days. For inclusion, the samples were stored in MMA1 solution with $1 \mathrm{w} \%$ of benzoyl peroxide for 3 days and in MMA1 solution with $2 \mathrm{w} \%$ of benzoyl peroxide for 3 days. The solidification took place in PTFE flat embedding molds covered by ACLAR film at $32{ }^{\circ} \mathrm{C}$ for $48 \mathrm{~h}$. The resin-embedded blocks were then trimmed and cut with a diamond knife in a Leica UC6 ultramicrotome. The 50-nm-thick transverse sections (i.e., normal to the long axis of the femur) were deposited on 200 mesh Cu TEM grids coated with lacey carbon.

\subsection{TEM data acquisition}


The measurements were performed using a JEOL 2100F FEG-TEM (Schottky ZrO/W field emission gun) operating at an accelerating voltage of $200 \mathrm{kV}$ and providing an electron beam focused to $2 \mathrm{~nm}$ in diameter at sample position. A camera was positioned in front of the TEM front window to collect diffraction patterns as a function of scanning position with a frame rate of $100 \mathrm{~Hz}$. The regions of interest were first selected in standard bright-field illumination (supplementary Fig. S3). The field of view for ACOM acquisition was chosen to be $400 \times 400 \mathrm{~nm}^{2}$ with a $10 \mathrm{~ms}$ acquisition time and a $2 \mathrm{~nm}$ step size. A sample-to-camera distance of $30 \mathrm{~cm}$ was chosen for all samples, except for the larger crystals treated at 800 and $1000{ }^{\circ} \mathrm{C}$, for which a camera length of $40 \mathrm{~cm}$ was used, applying a precession angle of $1.2^{\circ}$ at a frequency of $100 \mathrm{~Hz}$ in order to minimize dynamical effects [14]. Following distortion and camera length corrections, a virtual bright-field image was reconstructed numerically by selecting only the transmitted beam intensities.

\subsection{Radiation damage assessment}

No severe radiation damage was observed during ACOM-TEM data acquisition. This was assessed by independent bright-field acquisitions in the region close to the one scanned by ACOM-TEM for each heat-treatment temperature. These measurements were performed under the same conditions but with a smaller spot size of $0.7 \mathrm{~nm}$ (i.e. with a higher radiation dose) and were repeated 25 times to emphasize potential damage. Examples of bright-field images before the first and last $\left(25^{\text {th }}\right)$ frames are shown in Fig. S5 (supplementary information), showing very limited radiation damage.

\subsection{ACOM-TEM analysis}

The data analysis relies on the comparison between the electron diffraction patterns collected at every scan position and simulated patterns (templates) calculated for a given crystal structure in all possible orientations [14,23], thus allowing the reconstruction of crystal orientation maps (Fig. 1). The template matching was performed using the ASTAR software package from NanoMEGAS SPRL. In its native state, bone mineral is calcium phosphate close to a well-known hydroxyapatite, $\mathrm{Ca}_{10}\left(\mathrm{PO}_{4}\right)_{6}(\mathrm{OH})_{2}$, a subset of the widespread geological apatite minerals [8]. Hence, our initial model for the crystal structure is a hexagonal space group $\left(P 6_{3} / m\right)$ with 44 atoms per unit cell and lattice parameters of $a=9.417$ $\AA ; c=6.875 \AA[24]$. 


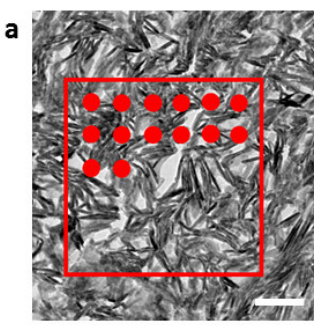

BF

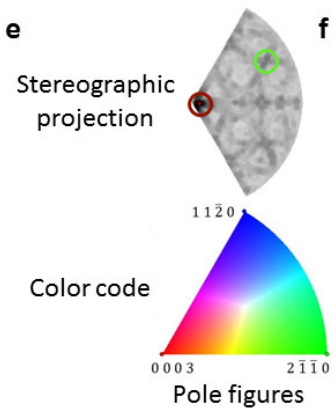

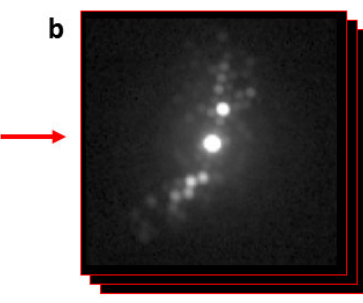

Set of diffractions

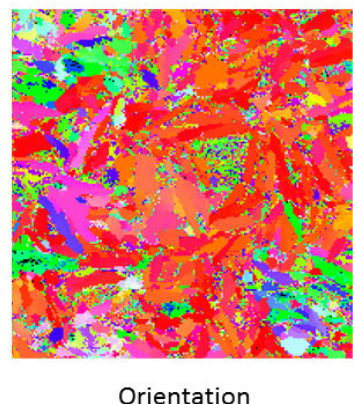

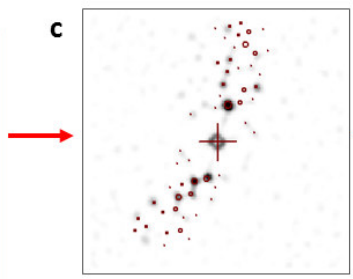

Fit

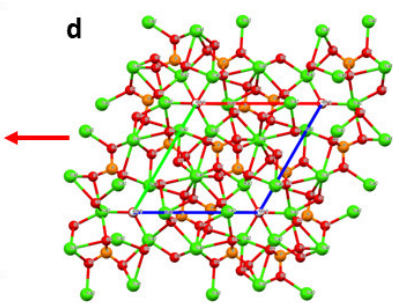

Hydroxyapatite template

Figure 1. Generalized scheme of ACOM-TEM acquisition and data interpretation:

(a) bright-field (BF) image of bone tissue with the illustration of the scan area (dots diameter is $2 \mathrm{~nm}$, enhanced for visibility), (b) recorded set of diffraction patterns, (c) example of fit using (d) the structure template for hydroxyapatite. (e) Fraction of stereographic projection with inverse pole figure color map, (f) orientation, (g) index and (h) reliability maps (high values appear brighter). Scale bar: $100 \mathrm{~nm}$

Every $i$-th acquired diffraction pattern collected at position $\left(x_{i}, y_{i}\right)$ was compared to the full set of templates through image correlation (template matching) and the best fit gave the most probable corresponding crystallographic orientation. This first result can thus be represented in the form of a color map representing the crystalline orientation (Fig. 1f). To assess the quality of the fit, a second map of the correlation index $Q_{i}$ can be used, defining $Q_{i}$ as:

$$
Q_{i}=\frac{\sum_{j=1}^{m} P\left(x_{j}, y_{j}\right) T_{i}\left(x_{j}, y_{j}\right)}{\sqrt{\sum_{j=1}^{m} P^{2}\left(x_{j}, y_{j}\right)} \sqrt{\sum_{j=1}^{m} T_{i}^{2}\left(x_{j}, y_{j}\right)}},
$$

where $P\left(x_{j}, y_{j}\right)$ is the intensity of measured diffraction patterns and $T_{i}(x, y)$ corresponds to the intensity in every $i$-th template. $Q i$ compares the intensities of the reflection contained in the diffraction pattern, denoted by the function $P(x, y)$, to the corresponding modeled intensities $T_{i}(x, y)$ in every $i$-th template in order to select the best match [23]. The degree of matching is represented in an 'index map' that plots the highest matching index at every location (Fig. $1 \mathrm{~g})$. This parameter therefore weights the degree of correlation between the acquired and 
simulated diffraction patterns. If more than one phase is expected to be present, several sets of templates can simultaneously be fitted to the data in order to recognize the best one. This allows constructing 'phase maps' in which each crystallographic phase is associated to a given color.

A critical aspect of the ACOM analysis is to judge the quality of the proposed crystal orientation. Indeed, it is worth emphasizing that the template matching algorithm always provides a solution, which requires evaluating the fidelity of the phase/orientation assignment, especially in the case of overlapping crystals. A reliability parameter $R_{i}$ was proposed to address this point. It is proportional to the ratio of the correlation indices for the two best solutions and is defined by:

$$
R_{i}=100\left(1-\frac{Q_{i 2}}{Q_{i 1}}\right)
$$

where $Q_{i 1}$ is the best solution (represented as a red circle on the stereographic projection in Fig. 1e) and $Q_{i 2}$ is the second best solution (shown with a green circle). Reliability values range between 0 (unsafe/black) and 100 (unique solution/white). In practice, a value above 15 is sufficient to ascertain the validity of the matching (Fig. 1h).

\section{Results}

\subsection{Individual nanocrystal visualization}

Bone nanocrystal orientation maps were derived for the set of heated bone samples (Fig. 2) with the corresponding collective orientations on the 0001 stereographic projection. A moderate increase in crystal size was observed below $800{ }^{\circ} \mathrm{C}$ followed by a rapid growth at higher temperatures with a crystal shape change from platelet to polyhedral.

An important advantage of ACOM is that the size and geometry measurements in bright-field TEM such as in Fig. 1a are generally performed on the whole image. In our case, the nanocrystals can exhibit a broad distribution of orientations, such that size estimation is impractical due to the platelet-shaped crystal geometry and leads to overestimated values as pointed out in earlier studies [25]. The additional visualization of the nanocrystal orientation therefore allows restricting the dimensional analysis to crystals in the same orientation (the same color-code). For example, crystals displayed in red in Fig.1f are oriented with their $c$ axis (longest axis) perpendicular to the scanning plane. The crystals displayed in green and blue have a $90^{\circ}$ misorientation from this particular zone axis. While the colors are representative of the crystal orientations, the overall difference between the ratio of red vs 
green and blue colors at different temperatures reflect the spatial coherence in the phase index and, thus, the level of heterogeneity within a particular sample or between different samples. This fact is confirmed by additional scans with larger fields of view acquired for each sample of the temperature series (supplementary information, Fig. S4).
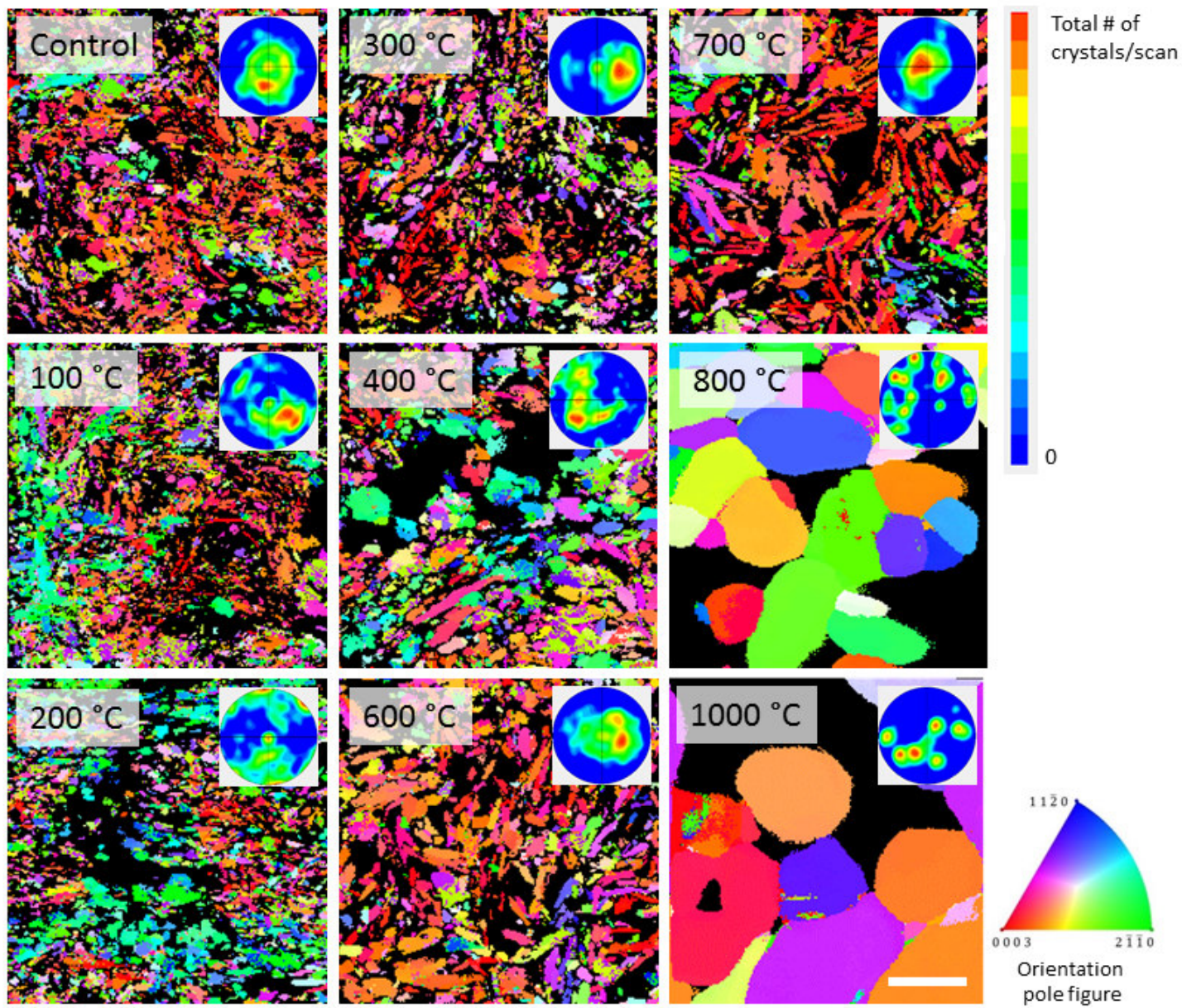

Figure 2. Mineral crystal orientation distribution in bone tissue as a function of temperature: orientation maps reconstructed from ACOM-TEM from control to $1000{ }^{\circ} \mathrm{C}$ samples with corresponding inverse pole figure color map (scale bar: $100 \mathrm{~nm}$ ). Inset: collective crystal orientations on 0001 stereographic projection with the color bar normalized to the total number of crystals per scan.

The crystallographic texture can be inferred from the stereographic projection along the 0001 direction showing that, below $800{ }^{\circ} \mathrm{C}$, most regions consist of crystals mainly aligned along the $c$-axis, which is in good agreement with other larger scale XRD studies [26]. At higher temperatures, we observed randomly oriented crystals resulting from a phase transition which nature will be discussed in a later section. 


\subsection{Bone mineral nanocrystal size estimation}

The nanocrystal size was measured assuming two models: platelet (anisotropic) for the low temperature (LT) phase below $750{ }^{\circ} \mathrm{C}$ and polyhedral (isotropic) for the high temperature (HT) phase above $750{ }^{\circ} \mathrm{C}$.

In the LT phase, the smallest platelet dimensions are obtained by line profiling (as displayed in Fig. 3a) of crystals having their $c$-axis aligned with the beam direction (represented by a red color). Crystal overlapping effects are not expected for this orientation as the long axis length of a platelet is comparable to the sample thickness. Therefore, the electron beam is predicted to pass through one crystal only, thus providing reliable size estimation.

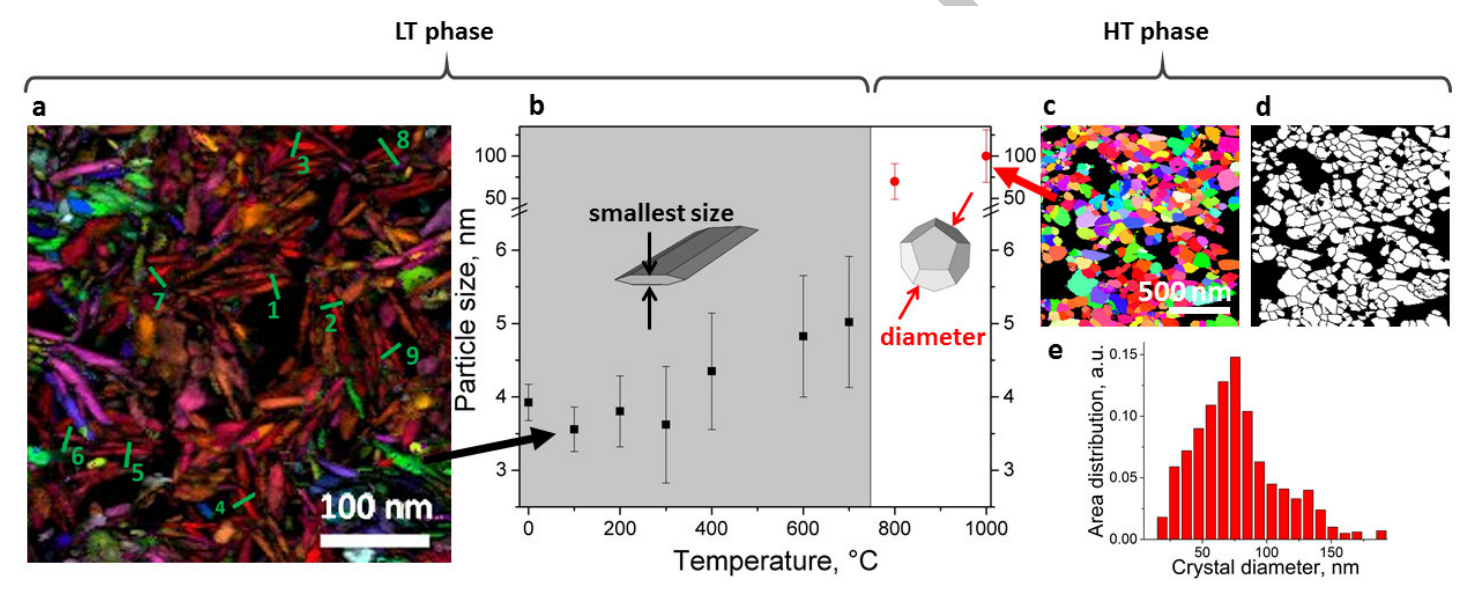

Figure 3. Bone mineral crystal size evolution with temperature: (a) an example of size measurement by line profiling along the $c$-axis of the platelet-shaped crystals for the LT regime (700 ${ }^{\circ} \mathrm{C}$ ); (b) average crystal size vs temperature (black - smallest crystal size, red - crystal diameter). Examples of polyhedral-shaped crystal diameter measurement for the HT regime $\left(800{ }^{\circ} \mathrm{C}\right)$ : $(\mathbf{c}, \mathbf{d})$ indicate orientation and grain boundary maps, respectively, and (e) represents the distribution of crystal diameters.

For the HT phase, the nanocrystal size was determined using a spherical approximation (crystal diameters) based on the definition of grain boundaries, i.e. the locations where the misorientation between two pixels at the orientation map (Fig. 3c) is higher than a user selected threshold value ( $5^{\circ}$ in the present case). An example of a grain boundary map for the $800{ }^{\circ} \mathrm{C}$ sample is shown in Fig. 3d. An average grain size is then estimated using a sphere 
diameter weighted by the grain's area in order to avoid misindexing from numerous small grains (mainly noise).

The grain size distribution was then obtained for the HT phase (Fig. 3e). According to the two described models for the nanocrystal sizes (summarized in Fig. 3b), we found that the smallest bone mineral particle size rises, on average, from $3.5 \mathrm{~nm}$ in the control state to 5.1 $\mathrm{nm}$ at $700{ }^{\circ} \mathrm{C}$. Subsequently, the average particle diameter dramatically increases upon further heating: up to $70 \mathrm{~nm}$ at $800{ }^{\circ} \mathrm{C}$ and $94 \mathrm{~nm}$ at $1000{ }^{\circ} \mathrm{C}$.

\subsection{Identification of the high temperature apatite phase}

While stoichiometric hydroxyapatite has a calcium-to-phosphate ratio of 1.67 , bone mineral is known to accommodate $\sim 7 \mathrm{wt} . \%$ of carbonate and numerous other ionic substitutions [8]. To test the sensitivity of ACOM-TEM to changes in stoichiometry and therefore of space group, we used the data set containing the largest grains $\left(1000{ }^{\circ} \mathrm{C}\right)$. We compared the fits obtained with the hydroxyapatite template against four template structures of different minerals occurring in nature with similar chemical composition and stoichiometry: alpha-tetra calcium phosphate (TCP) $\left(\alpha-\mathrm{Ca}_{3}\left(\mathrm{PO}_{4}\right)_{2}\right)\left(\right.$ space group - $\left.P 2_{1} / a\right)$, beta-Ca pyrophosphate $\left(\beta-\mathrm{Ca}_{2} \mathrm{P}_{2} \mathrm{O}_{7}\right)\left(P_{4}\right)$, tetra-calcium phosphate $\left(\mathrm{Ca}_{4}\left(\mathrm{PO}_{4}\right)_{2} \mathrm{O}\right)\left(P 2_{1}\right), \mathrm{CaO}$ $(F m \overline{3} m)$ and whitlockite $(R 3 c)$, which chemical compositions and structures are shown in Fig. 4a-e. Those phases were previously encountered in synthetic apatites subjected to heat treatments and could, therefore, be potential candidates for heated bone [27]. Since these minerals have different space groups, all possible orientations are described by different fractions of the stereographic projection allowed by symmetry, as shown by the color map shapes in Fig. 4.

Two criteria can be used to conclude that the hydroxyapatite template (Fig. 4a) provides the best solution: 1) the highest index value that characterizes the quality of the solution is nearly twice larger for hydroxyapatite than for other apatite structures; in addition, 2) a given particle is expected to be fitted with the same orientation if monocrystalline, resulting in a uniform color, which is only fulfilled for hydroxyapatite. This analysis provides a first proofof-concept that ACOM-TEM has a sufficient sensitivity to identify subtle variations of the crystal lattice that can be expected in highly disordered biological mineral structures, such as bone mineral nanocrystals. 


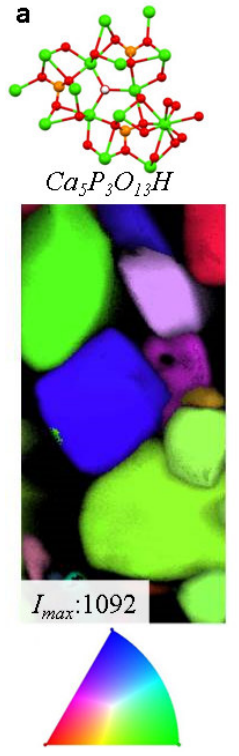

Hydroxyapatite

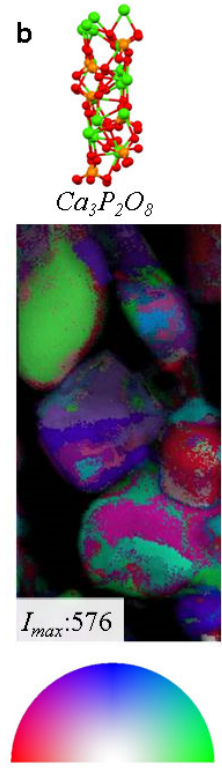

$\alpha-$ TCP

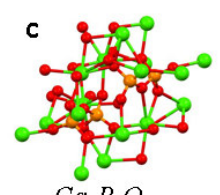

$\mathrm{Ca}_{2} \mathrm{P}_{4} \mathrm{O}_{7}$

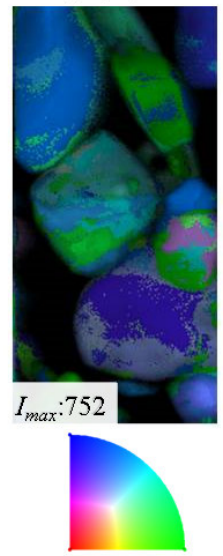

$\beta$-Ca pyrophosphate

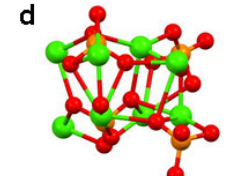

$\mathrm{Ca}_{4} \mathrm{P}_{2} \mathrm{O}_{9}$

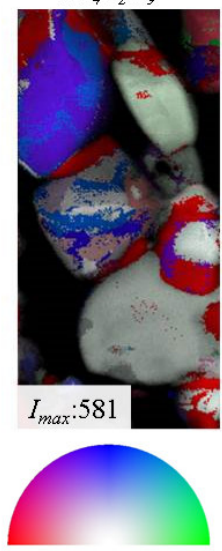

tetra-CP

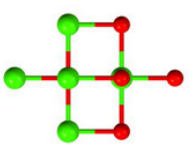

$\mathrm{CaO}$

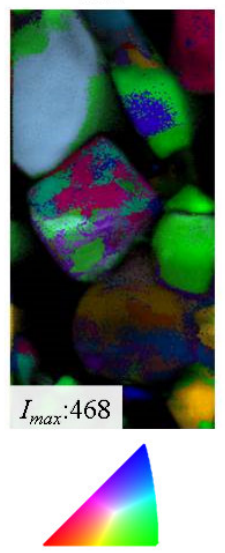

$\mathrm{CaO}$
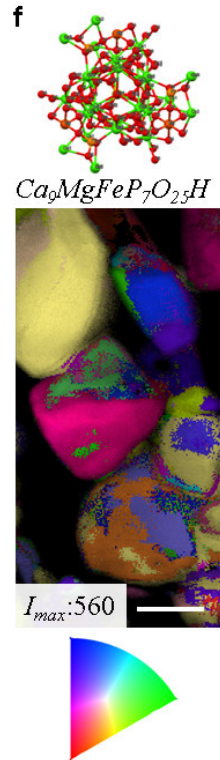

Whitlockite

Figure 4. Phase sensitivity: a-f, orientation maps for $100{ }^{\circ} \mathrm{C}$ heated bone data fitted with six apatite structures with corresponding inverse pole figure color maps. Maximum index values $\left(I_{\max }\right)$ are given for comparison. The hydroxyapatite structure produces the best fit indicated by the highest $I_{\max }$ value and homogeneous colors within single crystalsScale bar: $50 \mathrm{~nm}$.

Other apatite minerals which are not expected to be found in bone tissue but have close to hydroxyapatite stoichiometry and chemical composition such as brushite (space group Ia), monetite $(P \overline{1})$ and tuite $(R \overline{3} m)[28-31]$ were also used to test the ACOM-TEM sensitivity. I.e. if ACOM-TEM had resulted in an equal probability to find these phases, it would clearly have suggested a lack of precision of the method. The analysis shows that this is not the case, i.e. these phases did not allow describing bone data as well as hydroxyapatite (see Figure S5a-d in supplementary information).

\subsection{Space group: monoclinic or hexagonal ?}

A common issue in the identification of a hydroxyapatite phase at different temperatures

is the hypothesis of the existence of a hexagonal $\left(P 6_{3} / m\right)$ to monoclinic $(P 21 / b)$ phase transition above $T_{c r}=750{ }^{\circ} \mathrm{C}$. The corresponding structures are shown in Fig. 5a. However, such a transition was mainly predicted by theoretical models $[32,33]$ and was only observed in artificially synthesized hydroxyapatite [34-36]. From the theoretical point of view, the monoclinic hydroxyapatite structure is thermodynamically more stable than the hexagonal 
one. Nevertheless, the hexagonal phase allows an easier exchange of $\mathrm{OH}$-groups with other ions, which is necessary for bone tissue functions.

This issue was therefore addressed by matching the hydroxyapatite templates of the hexagonal [24] (Fig. 5b) and the monoclinic [37] (Fig. 5c) structures with the $1000{ }^{\circ} \mathrm{C}$ bone data set. The phase map in Fig. 5d represents the structure with the highest index at each scan point. Based on the index values, as well as on the uniform color-code criterion for single crystals, one can conclude that the hexagonal space group in bone mineral HT phase is more probable than the monoclinic one.

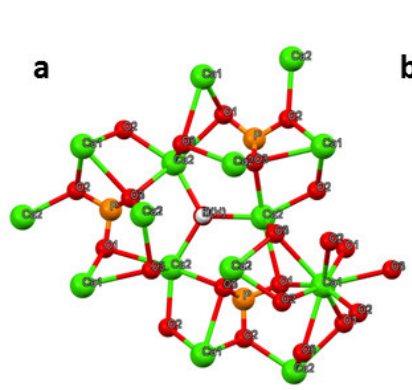

Hexagonal HAP

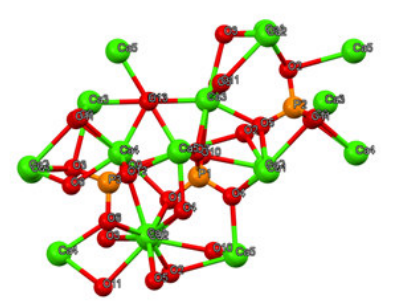

Monoclinic HAP
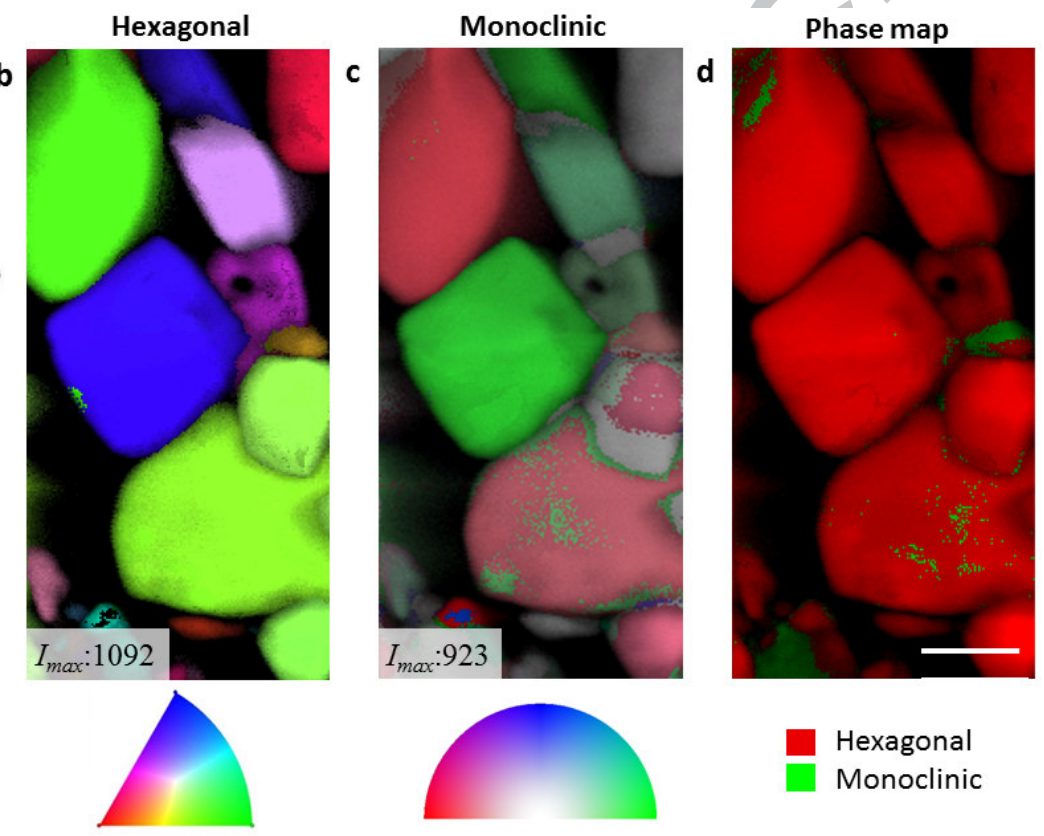

Figure 5. Hexagonal vs monoclinic symmetry: (a) crystal structures of hexagonal and monoclinic hydroxyapatite (view along $c$-axis); (b, c) corresponding orientation maps for the $1000{ }^{\circ} \mathrm{C}$ sample with color code and maximum index values; (d) phase map showing mainly the presence of hexagonal phase. Scale bar: $50 \mathrm{~nm}$.

\section{Discussion}

Our results provide a first demonstration that a structural analysis is possible at the single nanocrystal level within bone tissue using ACOM-TEM. This constitutes a valuable improvement combining the advantages of selected area electron diffraction (SAED) and TEM. While SAED produces a global diffraction pattern from all the nanocrystals probed by an electron beam defined by a micron sized aperture, ACOM-TEM allows a detailed structural analysis within a similar field of view. 
The use of the artificially heated bone model shows that the phase and orientation can be unambiguously determined for temperatures above $\sim 400{ }^{\circ} \mathrm{C}$ where the crystal size is $>5$ $\mathrm{nm}$ (Fig. 2). Even below this temperature, where the situation is less clear, since the scanning resolution given by the beam size $(\sim 2 \mathrm{~nm})$ is close to the actual nanocrystals size $(\sim 4-5 \mathrm{~nm})$, the observation of larger fields of view reveals the presence of coherently oriented domains in the control sample with characteristic sizes of $\sim 100-200 \mathrm{~nm}$ which are compatible with the diameter of collagen fibrils (supplementary information, Fig. S4). Such crystallographic information is not available from standard bright-field TEM and can be used to obtain more rigorous estimates of the nanocrystals dimensions. An important limitation of a bright-field TEM assessment of the thickness of the mineral platelets is that the nanocrystals can be sectioned in different orientation, hence resulting in an artificial broadening in projection [25]. These artefacts can be avoided using ACOM-TEM by only considering the platelets oriented on-edge, i.e. with the c-axis normal to the observation plane (Fig. 3). Similarly, depending on the thickness of the sample section, several nanocrystals may partly overlap, which further complicates the analysis. This case can be avoided by discarding the areas corresponding to a low correlation index and reliability parameters, i.e. to a poor structural refinement.

Our analysis reveals three stages of crystal growth upon heating: a first, moderate growth (from $~ 3.5$ to $5.1 \mathrm{~nm}$ ) between ambient temperature and $\sim 700{ }^{\circ} \mathrm{C}$, an order of magnitude increase in size (from $\sim 5.1$ to $70 \mathrm{~nm}$ ) between $700-800{ }^{\circ} \mathrm{C}$, followed by an additional growth (from 70 to $94 \mathrm{~nm}$ ) between $800-1000{ }^{\circ} \mathrm{C}$. This is in very good agreement with previous X-ray studies $[18,20,38]$. However, X-ray scattering provides average information from all crystals illuminated by the beam, while ACOM-TEM allows a precise mechanistic interpretation of the heating process. In particular, the appearance of new uncorrelated orientations at temperatures $>700{ }^{\circ} \mathrm{C}$ strongly suggests a recrystallization process by fusion-recrystallization of smaller grains. Interestingly, this process can qualitatively be observed from $400{ }^{\circ} \mathrm{C}$ onwards (i.e. following total collagen degradation) in Fig. 2, as the structure qualitatively becomes more heterogeneous (larger polydispersity in crystal sizes) and disordered. However, there is a sharp transition from platelets to polyhedral crystals between $700-800{ }^{\circ} \mathrm{C}$, clearly indicating a non-linear crystal growth.

One major difficulty in assessing the crystalline structure of bone is that the crystal chemistry is known to fluctuate, potentially giving rise to modulations of the intensity and breadth of the Bragg peaks which thus tend to overlap in XRD [39,40]. For the same reason, the precise interpretation of Raman and FTIR spectra is still a matter of debate after decades 
of studies [8]. Since the crystal structure is used as input in the ACOM-TEM analysis, such fine deviations to an ideal crystal structure could not be assessed reliably. Nevertheless, the analysis conducted with different templates shows that this method permits a reliable distinction between different phases on the basis of intrinsic quality metrics (correlation index) and extrinsic ones, e.g. the spatial coherence (color uniformity) of the phase and orientation determination.

This allowed, in particular, testing the hypothesis proposed in previous XRD studies that diffraction patterns of heated bone could be equally well indexed with a monoclinic space group instead of a hexagonal one. The lattice parameters of the two structures were found to be very close, with a $\beta$ angle close to $120^{\circ}$ for the monoclinic phase [38]. The main difference is that the length of the $b$-axis can fluctuate significantly from the $a$-axis in the monoclinic case (contrary to the hexagonal case where the $a$ - and $b$-axis are identical by definition). Thus, the difference between the two structures is relatively subtle but, in principle, a monoclinic structure should better account for a higher degree of crystallinity generated by heating, as demonstrated with synthetic apatites [34]. Our results conclusively show that even for samples treated at $1000{ }^{\circ} \mathrm{C}$, the mineral is better represented by a hexagonal structure. This was further confirmed by a close manual examination of the proposed solutions for a number of representative diffraction patterns (example in supplementary information, Fig. S6). Because the monoclinic phase is more representative of stoichiometric hydroxyapatite, the fact that bone mineral is better indexed by a hexagonal group implies that there is still a substantial degree of disorder in the crystal structure even for bone heated at high temperatures.

It is important to note that the ACOM setup can be readily implemented in standard existing TEM instruments and can therefore provide a close-to routine basis for biological, medical and archeological studies. Given the resolution level, we believe that ACOM-TEM could be advantageously exploited to analyze the interface layer (typically $<1 \mu \mathrm{m}$ ) between biomaterials and bone formed at the surface of implants, a critical aspect of osseointegration $[41,42]$. TEM was widely used to investigate the tissue structure at this interface $[43,44]$, but the collective mineral nanocrystals structure and organization was never analyzed. Similarly, in the biomedical field, severe pathological perturbations of mineral nanocrystals have been reported in many bone diseases, e.g. osteoporosis [45], osteogenesis imperfecta [46] and rickets [47], for which a detailed nanoscale description is still lacking [48]. Additionally, this method could also have a positive impact in the archaeological field, since diagenetic effects 
associated with long burial time of bone remains are known to affect the mineral ultrastructure in numerous ways, hence impacting the identification and conservation of bone artifacts [49]. Finally, it should be mentioned that ACOM-TEM would most likely benefit from more advanced sample preparation methods such as focused ion beam milling coupled to scanning electron microscopy (FIB-SEM) which has been shown to better preserve the tissue ultrastructure [50-52].

\section{Conclusion}

In the present work, we showed that both insights, the direct visualization of individual bone nanocrystals and structural information, can simultaneously be accessed using ACOMTEM analysis. The mineral nanocrystal orientation, crystallographic phase and symmetry can be quantified, even in biological samples such as bone tissue that are known to be very heterogeneous down to the nanoscale. Our analysis of a heated bone model points to a crystal growth by fusion and recrystallization mechanisms, starting from $400{ }^{\circ} \mathrm{C}$ onwards with a sharp transition between $700{ }^{\circ} \mathrm{C}$ and $800{ }^{\circ} \mathrm{C}$. By testing different phases corresponding to deviations from the hydroxyapatite stoichiometry as input for the structural refinement, we were able to assess the sensitivity of ACOM-TEM. We tested the hypothesis of a monoclinic space group attribution to the bone sample heated at $1000{ }^{\circ} \mathrm{C}$ and found that a hexagonal structure was more probable, suggesting the presence of crystalline defects even after heating at high temperatures. We therefore believe that ACOM-TEM could have a positive impact on applied research in biomaterials development, biomedical investigations of bone diseases and, possibly, analysis of archaeological bone remains.

\section{Acknowledgements}

The authors would like to acknowledge M. Morais from SIMaP for the support with the heating apparatus, D. Waroquy (ABAG, Grenoble, France) for providing the bovine samples, and the NanoBio-ICMG Platform (FR 2607, Grenoble) for granting access to the TEM sample preparation facility.

\section{Funding}

This work was supported by the Nanoscience Foundation (Ph.D. Grant of M.V. ${ }^{\ddagger}$ ). A part of this work also received the support from the European Union's Horizon 2020 research 
and innovation program under the Marie Skłodowska-Curie grant agreement No 701647 and SNSF grant No 200021L_169753.

\section{Author contributions}

M.V. , C.L.P. and J.L.P. prepared samples; M.V. $\ddagger$ E.F.R., M.V., M.P. and A.G. performed the research; A.G., M.P. and E.F.R. provided the financial support for the project; M.V. ${ }^{\ddagger}$ and E.F.R. analyzed data; M.V. ${ }^{\ddagger}$ and A.G. wrote the paper with contributions from all authors; A.G. and M.P. designed the research.

\section{Conflict of interest}

The authors declare no conflict of interest.

\section{References}

1. Weiner, S. \& Wagner, H. D. The Material Bone: Structure-Mechanical Function Relations. Annu. Rev. Mater. Sci. 28, 271-298 (1998).

2. Zimmermann, E. A., Schaible. E., Gludovatz B., Schmidt F. N., Riedel C., Krause M., Vettorazzi E., Acevedo C., Hahn M., Püschel K., Tang S., Amling M., Ritchie R. O. \& Busse B. Intrinsic mechanical behavior of femoral cortical bone in young, osteoporotic and bisphosphonate-treated individuals in low- and high energy fracture conditions. Sci. Rep. 6, 21072 (2016).

3. Matsushima, N. \& Hikichi, K. Age changes in the crystallinity of bone mineral and in the disorder of its crystal. Biochim. Biophys. Acta - Gen. Subj. 992, 155-159 (1989).

4. Boskey, A. L. Variations in bone mineral properties with age and disease. $J$. Musculoskelet. Neuronal Interact. 2, 532-534 (2002).

5. Granke, M., Gourrier, A., Rupin, F., Raum, K, Peyrin, F., Burghammer, M., Saïed, A. \& Laugier P. Microfibril Orientation Dominates the Microelastic Properties of Human Bone Tissue at the Lamellar Length Scale. PLoS One 8, (2013).

6. Chadefaux, C. \& Reiche, I. Archaeological Bone from Macro- to Nanoscale: HeatInduced Modifications at Low Temperatures. J. Nano Res. 8, 157-172 (2009). 
7. De Jong, W. Le substance minerale dans le os. Recl. Trav. Chim. Pays-Bas 45, 445450 (1926).

8. Wopenka, B. \& Pasteris, J. D. A mineralogical perspective on the apatite in bone. in Materials Science and Engineering C 25, 131-143 (2005).

9. Boivin, G., Chapuy, M.-C., Baud, C. A. \& Meunier, P. J. Fluoride content in human iliac bone: Results in controls, patients with fluorosis, and osteoporotic patients treated with fluoride. J. Bone Miner. Res. 3, 497-502 (1988).

10. Schroer, C. G., Kurapova, O., Patommel, J., Boye, P., Feldkamp, J., Lengeler, B., Burghammer, M., Riekel, C., Vincze, L., van der Hart, A., \& Küchler, M. Hard X-ray nanoprobe based on refractive x-ray lenses. Appl. Phys. Lett. 87, 124103 (2005).

11. Fratzl, P., Jakob, H. F., Rinnerthaler, S., Roschger, P. \& Klaushofer, K. PositionResolved Small-Angle X-ray Scattering of Complex Biological Materials. J. Appl. Crystallogr. 30, 765-769 (1997).

12. Paris, O. From diffraction to imaging: New avenues in studying hierarchical biological tissues with X-ray microbeams (Review). Biointerphases 3, FB16-FB26 (2008).

13. Xin, R., Leng, Y. \& Wang, N. HRTEM Study of the Mineral Phases in Human Cortical Bone. Adv. Eng. Mater. 12, B552-B557 (2010).

14. Rauch, E. F. \& Véron, M. Automated crystal orientation and phase mapping in TEM. Mater. Charact. 98, 1-9 (2014).

15. LeGeros, R. Z., Bonel, G. \& Legros, R. Types of 'H2O' in human enamel and in precipitated apatites. Calcif. Tissue Res. 26, 111-118 (1978).

16. Kubisz, L. \& Mielcarek, S. Differential scanning calorimetry and temperature dependence of electric conductivity in studies on denaturation process of bone collagen. in Journal of Non-Crystalline Solids 351, 2935-2939 (2005).

17. Etok, S. E., Valsami-Jones, E., Wess, T.J., Hiller, J. C., Maxwell, C. A., Rogers, K. D., Manning, D. A. C., White, M. L., Lopez-Capel, E., Collins, M. J., Buckley, M., Penkman, K. E. H. \& Woodgate, S. L. Structural and chemical changes of thermally treated bone apatite. in Journal of Materials Science 42, 9807-9816 (2007).

18. Rogers, K. D. \& Daniels, P. An X-ray diffraction study of the effects of heat treatment 
on bone mineral microstructure. Biomaterials 23, 2577-2585 (2002).

19. Hiller, J. C., Thompson, T. J. U., Evison, M. P., Chamberlain, A. T. \& Wess, T. J. Bone mineral change during experimental heating: An X-ray scattering investigation. Biomaterials 24, 5091-5097 (2003).

20. Piga, G., Malgosa, A., Thompson, T. J. U. \& Enzo, S. A new calibration of the XRD technique for the study of archaeological burned human remains. J. Archaeol. Sci. 35, 2171-2178 (2008).

21. Gourrier, A. Chadefaux, C., Lemaitre, E., Bellot-Gurlet, L., Reynolds, M., Burghammer, M., Plazanet, M., Boivin, G., Farlay, D., Bunk, O. \& Reiche, I.. Nanoscale modifications in the early heating stages of bone are heterogeneous at the microstructural scale. PLoS One 12, e0176179 (2017).

22. Greenwood, C., Rogers, K., Beckett, S. \& Clement, J. Initial observations of dynamically heated bone. Cryst. Res. Technol. 48, 1073-1082 (2013).

23. Rauch, E. F. \& Dupuy, L. Rapid spot diffraction patterns idendification through template matching. Arch. Metall. Mater. 55, 87-99 (2005).

24. Hughes, J. M., Cameron, M. \& Crowley, K. D. Structural variations in natural F, OH, and $\mathrm{Cl}$ apatites. Am. Mineral. 74, 870-876 (1989).

25. Ziv, V. \& Weiner, S. Bone Crystal Sizes: A Comparison of Transmission Electron Microscopic and X-Ray Diffraction Line Width Broadening Techniques. Connect. Tissue Res. 30, 165-175 (1994).

26. Voltolini, M., Wenk, H. R., Gomez Barreiro, J. \& Agarwal, S. C. Hydroxylapatite lattice preferred orientation in bone: A study of macaque, human and bovine samples. J. Appl. Crystallogr. 44, 928-934 (2011).

27. Berzina-Cimdina, L. \& Borodajenko, N. Research of Calcium Phosphates Using Fourier Transform Infrared Spectroscopy. in Infrared Spectroscopy - Materials Science, Engineering and Technology (InTech, 2012). doi:10.5772/36942

28. Schofield, P. F., Knight, K. S. \& van der Houwen, J. A. M. The role of hydrogen bonding in the thermal expansion and dehydration of brushite, di-calcium phosphate dihydrate. Phys. Chem. Miner. 31, 606-626 (2004). 
29. Catti, M., Ferraris, G. \& Filhol, A. Hydrogen bonding in the crystalline state. CaHPO4 (monetite), P1 or P1? A novel neutron diffraction study. Acta Crystallogr. Sect. B Struct. Crystallogr. Cryst. Chem. 33, 1223-1229 (1977).

30. Sugiyama, K. \& Tokonami, M. Structure and crystal chemistry of a dense polymorph of tricalcium phosphate $\mathrm{Ca} 3$ (PO4)2: A host to accommodate large lithophile elements in the earth's mantle. Phys. Chem. Miner. 15, 125-130 (1987).

31. Calvo C, G. R. The crystal structure of whitlockite from the Palermo quarry. Am. Mineral. 60, 120-133 (1975).

32. Slepko, A. \& Demkov, A. A. Hydroxyapatite: Vibrational spectra and monoclinic to hexagonal phase transition. J. Appl. Phys. 117, 74701 (2015).

33. Corno, M., Busco, C., Civalleri, B. \& Ugliengo, P. Periodic ab initio study of structural and vibrational features of hexagonal hydroxyapatite Ca10(PO4)6(OH)2. Phys. Chem. Chem. Phys. 8, 2464-2472 (2006).

34. Ma, G. \& Liu, X. Y. Hydroxyapatite: Hexagonal or monoclinic? Cryst. Growth Des. 9, 2991-2994 (2009).

35. Ikoma, T., Yamazaki, A., Nakamura, S. \& Masaru, A. Phase Transition of Monoclinic Hydroxyapatite. Netsu Sokutei 25, 141-149 (1998).

36. Suda, H., Yashima, M., Kakihana, M. \& Al., E. Monoclinic - Hexagonal Phase Transition in Hydroxyapatite Studied by X-ray Powder Diffraction and Differential Scanning Calorimeter Techniques. J. Phys. Chem. 99, 6752-6754 (1995).

37. Elliott, J. C., Mackie, P. E. \& Young, R. a. Monoclinic hydroxyapatite. Science 180, 1055-1057 (1973).

38. Piga, G., Solinas, C., Thompson, T. J. U., Brunetti, A., Malgosa, A. \& Enzo, S. Is Xray diffraction able to distinguish between animal and human bones? J. Archaeol. Sci. 40, 778-785 (2013).

39. Posner, A. S. Crystal chemistry of bone mineral. Physiol. Rev. 49, 760-792 (1969).

40. Sakae, T., Nakada, H. \& John P. LeGeros. Historical Review of Biological Apatite Crystallography. J. Hard Tissue Biol. 24, 111-122 (2015).

41. Davies, J. E. Bone bonding at natural and biomaterial surfaces. Biomaterials 28, 5058- 
5067 (2007).

42. LeGeros, R. Z. Calcium phosphate-based osteoinductive materials. Chem. Rev. 108, 4742-4753 (2008).

43. Grandfield, K., Palmquist, A. \& Engqvist, H. High-resolution three-dimensional probes of biomaterials and their interfaces. Phil. Trans. R. Soc. A 370, 1337-1351 (2012).

44. Palmquist, A. Grandfield, K., Norlindh, B., Mattsson, T., Brånemark, R. \& Thomsen, P. Bone--titanium oxide interface in humans revealed by transmission electron microscopy and electron tomography. J. R. Soc. Interface 420 (2011).

45. Rubin, M. A. Jasiuk, I., Taylor, J., Rubin, J., Ganey, T. \& Apkarian, R. P.. TEM analysis of the nanostructure of normal and osteoporotic human trabecular bone. Bone 33, 270-282 (2003).

46. Fratzl-Zelman, N. Schmidt, I., Roschger, P., Roschger, A., Glorieux, F. H., Klaushofer, K., Wagermaier, W., Rauch, F. \& Fratzl, P. Unique micro-and nano-scale mineralization pattern of human osteogenesis imperfecta type VI bone. Bone 73, 233 $241(2015)$.

47. Karunaratne, A. Esapa, C. R., Hiller, J., Boyde, A., Head, R., Bassett, J. H., Terrill, N. J., Williams, G. R., Brown, M. A., Croucher, P. I., Brown, S. D., Cox, R. D., Barber, A. H., Thakker, R. V. \& Gupta, H. S. Significant deterioration in nanomechanical quality occurs through incomplete extrafibrillar mineralization in rachitic bone: Evidence from in-situ synchrotron X-ray scattering and backscattered electron imaging. J. Bone Miner. Res. 27, 876-890 (2012).

48. Gourrier, A., Li, C., Siegel, S., Paris, O., Roschger, P.,Klaushofer, K. \& Fratzl, P. Scanning small-angle X-ray scattering analysis of the size and organization of the mineral nanoparticles in fluorotic bone using a stack of cards model. J. Appl. Crystallogr. 43, 1385-1392 (2010).

49. Reiche, I., Vignaud, C. \& Menu, M. The crystallinity of ancient bone and dentine: new insights by transmission electron microscopy. Archaeometry 44, 447-459 (2002).

50. Jantou, V., Turmaine, M., West, G., Horton, M. \&McComb, D. Focused ion beam milling and ultramicrotomy of mineralised ivory dentine for analytical transmission electron microscopy, Micron 40, 495-501 (2009). 
51. McNally, E.A., Schwarcz, H.P., Botton, G.A. \& Arsenault, A.L. A Model for the Ultrastructure of Bone Based on Electron Microscopy of Ion-Milled Sections, PLoS One 7, e29258 (2012).

52. Reznikov, N., Shahar, R. \& Weiner, S. Three-dimensional structure of human lamellar bone: the presence of two different materials and new insights into the hierarchical organization, Bone 59, 93-104 (2014). 

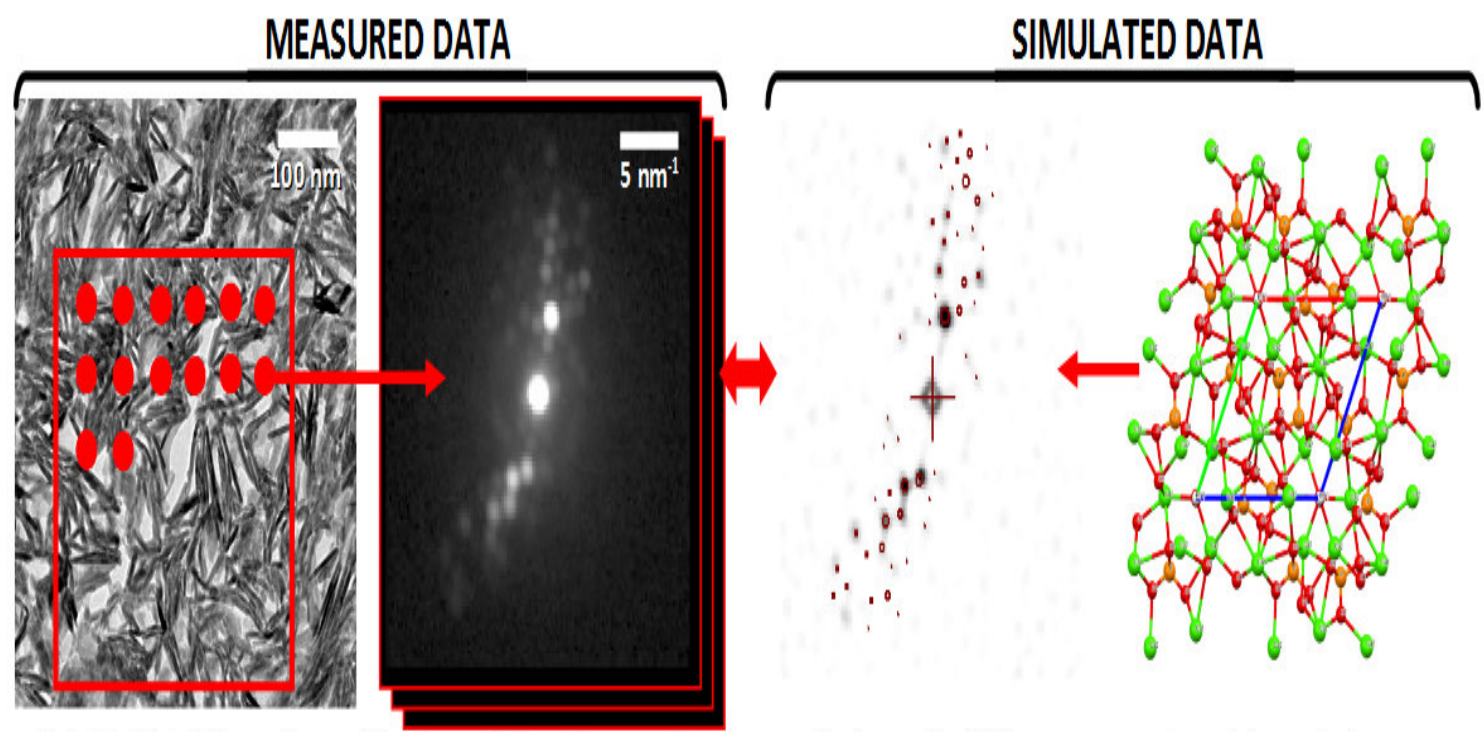

Bright Field Overview

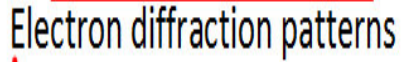

Automatic fitting

Input template

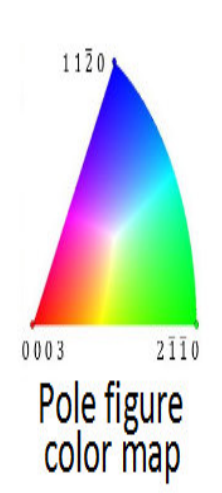

\section{ACOM-TEM}
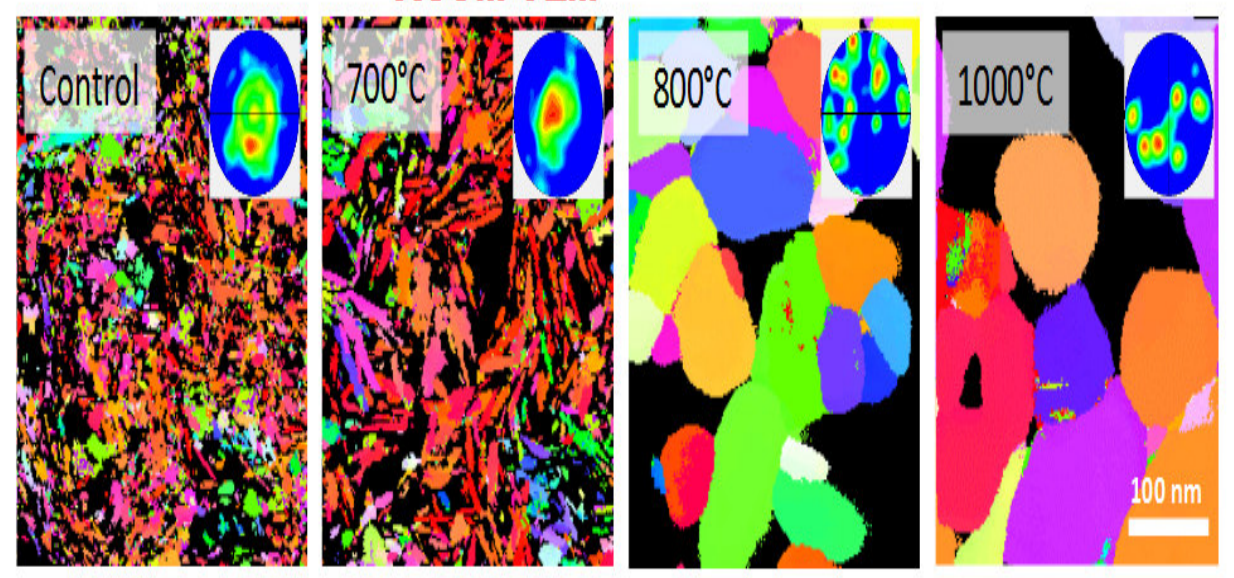